Invest. Pens. Crit. (ISSN 1812-3864; eISSN 2644-4119)

Vol. 9, No. 2, Mayo - Agosto 2021. pp. 62 -69

DOI: https://doi.org/10.37387/ipc.v9i2.235

Ensayo

\title{
Los jóvenes y la identidad del trabajo
}

\section{Youth and work identity}

\author{
Ida Graciela Gálvez Amores ${ }^{1 *}$ \\ ${ }^{1}$ Becaria de la Maestría en Ciencias Sociales con énfasis en Teorías y Métodos de investigación - SENACYT - Universidad de Panamá. \\ Centro de Investigaciones Científicas de Ciencias Sociales CENICS, Panamá.
}

*Autor para correspondencia: idagracielagalvez@gmail.com

Recibido: 04 de enero de 2021. Aceptado: 15 de marzo de 2021

\section{Resumen}

En el presente artículo se incluye una revisión del concepto de identidad y cómo esta se construye desde una perspectiva multidimensional en los distintos contextos en los que se incluyen las personas en su día a día. Específicamente se realiza el enfoque hacia el grupo etario juvenil, el cual vive situaciones considerables de precariedad y desigualdad en los ambientes laborales y sociales a raíz de los estereotipos y dinámicas que se han conformado en la sociedad actual. Esto se coloca como un asunto de importancia que incluye distintas esferas del desarrollo humano, ya que los jóvenes han consolidado su identidad desde la adolescencia con influencia de las situaciones que viven a nivel individual, político y social. Se presenta una síntesis de la situación laboral de los jóvenes en Panamá y se brinda una mirada al concepto de identidad laboral, el cual se toma como un sistema de creencias que se forma a partir del trabajo que se realiza y que tiene una influencia en la satisfacción laboral, en la manera en la que se configuran distintos movimientos sociales y en la autovalía que tienen los jóvenes con respecto a su productividad y a su capacidad para transformar. Es imprescindible la consideración de las variables psicosociales en el estudio de los fenómenos sociales en el país, ya que ellos determinan en gran medida el actuar de los grupos humanos y tienen un impacto importante en los engranajes que modifican las situaciones que tienen lugar en el país y a nivel global en la gestión de políticas públicas y desarrollo social.

Palabras clave: identidad, trabajo, jóvenes, sociedad, identidad laboral.

\begin{abstract}
The article includes a review of the concept of identity and its construction from a multidimensional perspective. Analyze the different contexts in which people are included in their daily lives. Specifically, the focus is made on the youth age group, which lives considerable precariousness and inequality in work and social environments because of the stereotypes and dynamics formed in today's society. This is placed as a matter of importance that includes different spheres of human development since young people have consolidated their identity since adolescence, influenced by their situations at an individual, political, and social level. A synthesis of the employment situation of young people in Panama is presented, and a look is given at the concept of work identity.
\end{abstract}


This is taken as a belief system formed from the work done and that influences job satisfaction, how different social movements are configured, and the self-worth that young people have concerning their productivity and their capacity to transform. Consideration of psychosocial variables is essential in the study of social phenomena in the country since they largely determine human groups' actions and have an essential impact on the mechanisms that modify the situations that take place in the country. And at a global level in the management of public policies and social development.

Keywords: identity, work, youth, society, work identity.

\section{Introducción}

El concepto de identidad se ha utilizado regularmente para hablar acerca de las características personales de un individuo y la forma en la que se ha constituido con respecto a la percepción que tiene de sí mismo. No obstante, la referencia de este concepto con respecto al trabajo ha venido dada por la introducción de variables psicológicas y la subjetivación de los individuos en diversos contextos, especialmente el laboral (MorenoHurtado, Arévalo, Martínez-Patiño, Martínez-Beltrán y Vesga-Rodríguez, 2018). Por otro lado, el estudio de la identidad se ha adentrado en una perspectiva colectiva y social, en donde se estructura a un sujeto que se forma a sí mismo a partir de las personas con las que interactúa (Ruvalcaba-Coyaso, Uribe Alvarado y Gutiérrez García, 2011).

Dentro de la psicología del trabajo, la identidad se ha colocado como objeto de estudio que responde a la necesidad de entender modelos de liderazgo gerenciales y elementos que promuevan una mejor satisfacción laboral. Además de que ha introducido diferenciaciones novedosas en materia de trabajo, como por ejemplo la distancia que existe entre las tareas que debe realizar un trabajador y la voluntad real que tiene para llevarlas a cabo (Pulido-Martínez, 2012). De esta forma es posible observar cómo se realiza una síntesis entre las variables situaciones y las variables personales que giraban en torno al concepto de trabajo, en donde el trabajador en materia científica deja de ser una máquina de producción y se convierte en un ser que tiene múltiples determinaciones y dimensiones a tomar en cuenta.

Con respecto a lo teórico referido a lo real, se considera que el desarrollo con respecto a los factores incidentes en el proceso del trabajo no ha respondido completamente a la situación actual que se vive con respecto a las relaciones y realidades laborales; ya que la transformación de los contextos y legislaciones laborales ha sido muy rápida en contraste con el desarrollo teórico existente (Moreno-Hurtado et. al, 2018). Principalmente, es por esta razón que se considera imperante tratar el tema de la identidad referida al trabajo en cuanto a la información científica existente que incluye a un grupo socialmente vulnerable como lo es la juventud.

En términos generales, la juventud se configura como un grupo que tiene desventajas en la esfera laboral en términos de obtención del trabajo, percepción de adecuación dentro de este y transiciones en el espacio de trabajo. De acuerdo con la Organización Internacional del Trabajo (2017) existe una situación de desafío con respecto al empleo juvenil, ya que la tasa de desempleo es tres veces más alta que la de los adultos y 2 de cada 5 jóvenes económicamente activos se encuentran sin trabajo. Esto consolida la importancia de revisar la información que existe con respecto a elementos personales de los jóvenes con respecto al trabajo con el objetivo de generar distintas hipótesis generadoras de información que permita superar este difícil desafío.

Esta revisión busca incluir algunas consideraciones científicas con respecto al concepto de identidad, describir brevemente la importancia del trabajo a nivel psicológico y social y, finalmente, resaltar la importancia del trabajo en la identidad de los sujetos jóvenes como un ente social que forma estructuras psíquicas referidas al entorno y tiene un impacto importante en el desenvolvimiento del joven primeramente como ciudadano y luego como trabajador productivo. 


\section{El concepto de identidad}

El concepto de identidad es uno de los que ha mantenido en mayor medida el vínculo existente entre la Psicología y las Ciencias Sociales; siendo que mantiene componentes psicológicos como la personalidad o el autoconcepto y componentes sociales de colectividad. Es por lo anterior que el concepto de identidad ha mantenido en los últimos años un corte psicosocial (Ovejero, 2015).

Si bien para la mayoría de los científicos puede existir una idea general de lo que es la identidad, el concepto encuentra distintas definiciones de acuerdo el área en el que se utiliza; además de que mantiene un desarrollo histórico distinto desde la perspectiva sociológica y la teoría psicológica.

En cuanto a una perspectiva sociológica, es posible observar las discusiones en torno a la identidad en algunos planteamientos como los de la escuela marxista y la escuela inglesa. En el marxismo la identidad tiene un vínculo profundo con la clase, en donde el no tener consciencia de clase lleva a la enajenación, mientras el tenerla crea una situación de "clase para sí". Por otro lado, para la escuela inglesa, la clase no existe en sí misma, sino que viene dada por experiencias comunes de un grupo y toma en cuenta considerablemente la subjetividad de los actores involucrados (Quinteros, 2011).

Ambas perspectivas desde una visión estrictamente sociológica incluyen la clase como determinante de la identidad y de la mirada que la persona tiene con respecto a lo que es y a lo que va a ser. Sin embargo, desde la Psicología es posible encontrar definiciones de identidad que incluyen otros elementos esenciales para comprender el concepto de identidad dentro del ambiente laboral.

En primer lugar, presentamos la definición de Burke y Stets (2009), quienes afirman que la identidad es un

"Conjunto de significados que define quién es uno cuando desempeña un rol específico en la sociedad, es miembro de un grupo particular, o afirma características particulares que lo definen como persona única".

Con una definición que incluye aspectos similares, Páramo (2008), define la identidad como las características que posee una persona y a través de las cuales es conocido. En su definición incluye que la identidad se forma a partir de las interacciones que mantenemos con otras personas y que determina considerablemente la manera en la que nos desenvolvemos en distintos contextos. Acorde a esta definición es posible mencionar la concepción que tuvo Erikson (citado en: Berzonsky, 1992) con respecto a la identidad, en donde la coloca como un hito más dentro del desarrollo evolutivo; es decir, una entidad que tiene su consolidación en la adolescencia y que, más allá de formar de promover la individualidad de la persona, le brinda las herramientas necesarias para formar parte de un engranaje social (Ruvalcaba-Coyaso, Uribe Alvarado y Gutiérrez García, 2011).

Si observamos las definiciones presentadas es posible identificar elementos comunes que vale la pena tener en consideración. En primer lugar, la identidad hace referencia a un proceso individual que se sirve de las experiencias externas para su formación y desarrollo. En segundo lugar, el proceso de formación de identidad y la identidad en sí misma, de acuerdo con las definiciones propuestas, tienen una incidencia directa con la participación social y los ambientes en los que se desarrolla la persona; siendo uno de estos ambientes principales el trabajo.

\section{El trabajo para los jóvenes}

El trabajo se coloca como un elemento principal en la inclusión social de las personas a nivel general. La socialización que se genera en el trabajo tiene como resultado diferentes estructuras de relación con respecto a grupos humanos y la generación de distintos valores y creencias con respecto a las actividades laborales (Gracia, Salanova y González, 1996). Si bien esta socialización ocurre en todas las cohortes generacionales, los jóvenes suponen un grupo de interés, ya que mantienen características particulares en relación con las oportunidades laborales, la retención de empleo y la validación dentro de los puestos de trabajo. 
En cuanto a la empleabilidad, encontramos que los jóvenes viven desigualdades considerables en cuanto a obtener un empleo formal en comparación con otros grupos (Sánchez-Castañeda, 2014). De acuerdo con la Organización Internacional del Trabajo (2020), esto puede deberse a que existen muchos procesos que han sido recientemente automatizados, hay limitaciones importantes en la formación profesional y hay escasez de empleo que se corresponda con la tendencia que se ha desarrollado con relación a la formación académica. Por otra parte, en los últimos años se ha observado un aumento en la educación superior de algunas áreas, lo que hace que la oferta sea superior a la demanda y los salarios se vean reducidos e insuficientes con respecto a la realidad económica mundial.

Además de las situaciones contextuales a las que se ven enfrentados los jóvenes en todas partes del mundo, existen factores individuales que pueden incidir en la manera en la que la juventud percibe las actividades laborales, lo que a su vez tiene un impacto en la ejecución de estas. La manera en la que el joven observa el mercado laboral va a determinar su interés en este, en donde se incluyen elementos como los contactos, las posibilidades reales de trabajo y la eficacia autopercibida vinculada a la manera en la que pueden llevar a cabo una actividad determinada (Pérez, Castro y Cubo, 2009). Desde otra perspectiva, observamos que las tendencias generacionales en cuanto a la personalidad y los modos de ejecución han pasado por una evolución consistente, en donde es posible observar la preferencia de los jóvenes por esquemas laborales que tengan mayor flexibilidad en cuanto a las tareas, ubicación y normativas dentro del lugar de trabajo; esto sin dejar a un lado que siguen orientados hacia un trabajo y horarios fijos y con cierto grado de formalidad (Freire, 2009).

Es importante acercarse al estudio de la posición de los jóvenes dentro de la dinámica laboral desde la premisa de que ambos elementos, los jóvenes y el trabajo, mantienen estructuras internas complejas y se afectan de forma bidireccional y simultánea. En otras palabras, el ambiente y las tareas laborales tienen un efecto directo en la formación del significado del trabajo de los jóvenes y a su vez los jóvenes generan transformaciones dentro de sus espacios de trabajo a través de sus características individuales y sociales como grupo con características particulares (Gracia, Salanova y González, 1996).

\section{Situación laboral de los jóvenes en Panamá}

A lo largo de los últimos años, se ha dado en Panamá una situación progresiva de dificultad económica, lo que ha aumentado el desempleo y la pobreza en distintos sectores del país. Los jóvenes, como grupo discriminado y con pocas oportunidades a nivel educativo y de acceso a trabajos formales, han sido uno de los grupos más afectados por estos cambios desde el año 2017 (Instituto de Estudios del Trabajo, 2019).

El informe emitido por la Organización Internacional del Trabajo en el 2017 habla acerca de los jóvenes que no estudian ni trabajan, los "ninis", quienes se encuentran en un estado precario en cuanto a su experiencia académica y a características demográficas específicas como el ser mujer (Ministerio de Trabajo, 2019).

Es notable las dificultades por las que pasan los jóvenes en Panamá con respecto al trabajo en diferentes esferas, siendo que en el grupo etario de entre los 15 y 24 años, para el 2017 únicamente el $61 \%$ mantenía un trabajo formal que cotizara seguro social (Instituto de Estudios del Trabajo, 2019). Esta realidad apunta hacia una juventud que está teniendo dificultades para obtener trabajo o para estar en una posición formal que incluya todas las prestaciones laborales a las que tienen derecho todos los ciudadanos y se han venido omitiendo producto de la explotación y precarización laboral disfrazada de modernidad e independencia.

Además de la dificultad para obtener un empleo, en Panamá se observa una dificultad notable en los jóvenes para mantenerlo, ya que son de los primeros en ser retirados de los puestos al tener que hacer reducciones de personal o al pasar por alteraciones económicas empresariales como las que ha causado la pandemia del COVID-19. Fenómeno que, según la Organización Internacional del Trabajo (2020), afectará en mayor medida a los jóvenes, ya que muchos no han tenido la oportunidad de continuar su educación básica y es probable que abandonen la escuela e incurran en trabajos informales o similares.

La situación es tan grave para la juventud que 1 de cada 6 jóvenes ha perdido el trabajo desde el inicio de la pandemia y, por esta razón, es necesario pensar en medidas de contingencia que permitan a los grupos de 
jóvenes obtener oportunidades dignas de trabajo y de educación que responda a las necesidades del país (Núñez, 2020).

Si bien el desempleo se ha colocado como una problemática en todos los grupos, se considera imprescindible colocar especial atención en los jóvenes y a la vinculación que tiene la situación dificultosa que viven con respecto al trabajo y la educación, ya que el sistema educativo pareciera ser el mayor gestor de las herramientas con las que deben contar los jóvenes para su desempeño en diferentes áreas laborales y el que tiene la oportunidad de reforzar un sistema de creencias que promueva los movimientos sociales y la consciencia de derechos ciudadanos en los jóvenes panameños.

\section{Identidad del trabajo}

La identidad se coloca como una entidad compleja, la cual pasa por diferentes procesos a lo largo de la vida y encuentra su consolidación en la fase de la adolescencia (Zacarés, Iborra, Tomás y Serra, 2009). Si bien la identidad es una construcción interna vinculada al autoconcepto y a la autovalía, tiene una estrecha relación con los sucesos contextuales del individuo y con la manera en la que se comprende su existencia en el mundo desde las actividades que realiza.

De acuerdo con la teoría del desarrollo de Erik Erikson, la formación de la identidad tiene que ver con el interés por el aprendizaje y la vivencia de nuevas experiencias; es un proceso que tiene lugar en la pubertad y más allá de tener eco en los factores individuales, se vincula a cómo el joven se incluye en la dinámica social (Ruvalcaba-Coyaso, Uribe y Gutiérrez, 2011). Por otro lado, autores como Millón (1998) colocan la identidad como un proceso automático y fuera del consciente que produce acciones específicas en el individuo y es prácticamente inamovible. Como es posible concluir a partir de ambas definiciones, existen teóricos que colocan el constructor de identidad como una entidad sujeta a influencias ambientales, mientras otros consideran que la identidad no pasa por cambios significativos y es relativamente estable durante toda la vida.

Esta discusión teórica con respecto al desarrollo e influencia de la identidad contiene su solución en la medida en la que se entienda al individuo humano inserto dentro de un contexto social. Es por esto por lo que, desde lo social, encontramos imprescindible observar a la identidad como un elemento estable pero transformable y sujeto a modificaciones a partir de las experiencias de vida y de los hitos transicionales, siendo uno de ellos el trabajo. No es producente estudiar y observar la identidad desde una perspectiva reduccionista, ya que la formación de esta se ve impactada por distintos elementos vivenciales, ambientales y sociales desde la infancia.

Tomando en consideración que la identidad toma elementos del ambiente y de terceros para configurarse dentro del individuo, es esperado que lo laboral juegue un papel considerable en la identidad de los jóvenes, ya que el trabajo se ubica como la actividad que subjetiviza a las personas dentro de la sociedad y la que le otorga un lugar específico dentro de la dinámica social de producción. Lo anterior cobra sentido a partir de un sistema que históricamente ha ido evolucionando debido al modelo capitalista en donde la producción cuenta con una aprobación generalizada y con una validación impuesta de la tarea que se lleva a cabo como trabajo.

Es por lo anterior que se consolida el concepto de identidad en el trabajo, identidad profesional o identidad organizacional, el cual hace referencia a un sistema de creencias y percepciones que rodean la actividad laboral que lleva a cabo un individuo. La construcción de esta identidad diferencia a un grupo de trabajadores de otro y moldea de distintas formas su manera de acercarse al mundo social, la forma en la que se relacionan y su ideología dominante (Téllez, 2002).

La identidad del trabajo ha sido estudiada como variable personal-social y también como estructura de la que depende la solidez y la percepción positiva que se tiene de una organización (Duque y Carvajal, 2015). Es necesario reconocer esta entidad conceptual como un proceso que tiene distintas aristas y que influye en el desempeño del trabajador y también en la satisfacción que tiene con relación a su trabajo; la cual no solamente responde a reforzadores externos y físicos sino a recompensas no tangibles y sentimientos que remiten a un sentido de autovalía y aporte a la sociedad en la que se vive. 
El estudio de la identidad social tomando en cuenta las variables contextuales y personales supone una tarea dificultosa en la cual se pone en juego la rigurosidad científica y la supuesta objetividad necesaria para ejercer métodos respetables. No obstante, al estudiar fenómenos intrínsecamente humanos es necesario tomar la subjetividad como una oportunidad de establecer parámetros científicos novedosos y tener una idea global de lo que viven distintos grupos humanos desde la forma en la que se identifican y el impacto de esto en el entramado social.

La complejidad y los diferentes factores que subyacen al concepto de identidad del trabajo hacen a este fenómeno una variable de estudio prometedora la cual permitirá gestionar políticas de acción y transformación social que respondan a las problemáticas laborales que se han venido desarrollando en los últimos años como lo es el desempleo juvenil y las condiciones de trabajo en nuevas modalidades de empleo como el teletrabajo.

\section{Conclusiones}

En este artículo de revisión ha sido posible mencionar algunos de los puntos esenciales al momento de hablar del concepto de identidad y, específicamente de la misma dentro del ámbito laboral. A partir de la situación que vive el mundo entero y, específicamente Panamá, en cuanto a la empleabilidad juvenil y las condiciones de trabajo, se considera imprescindible el desarrollo de investigaciones empíricas que permitan otorgar datos cuantitativos y cualitativos que brinden una perspectiva científica acerca de la realidad juvenil y el imaginario que se construye alrededor del trabajo.

Específicamente en Panamá, encontramos que cerca de la mitad de los jóvenes carecen de un trabajo formal que les brinde garantías y derechos laborales justos y que permitan la colaboración individuo-Estado para el sostenimiento de los sistemas educativos y de salud, además de necesidades posteriores individuales como lo es la jubilación. Las razones detrás de esta problemática laboral en jóvenes las encontramos en muchas ocasiones en los estereotipos que existen con respecto a la juventud, en donde se asume que la falta de experiencia tiene como consecuencia un trabajo deficiente y en donde el joven es la primera opción al realizar recortes presupuestarios o cambios de personal.

Tomando en cuenta la creciente necesidad juvenil y el surgimiento de nuevas tecnologías y novedosos campos de trabajo en Panamá, es imprescindible la adaptación de los lugares de trabajo a las necesidades que presentan los jóvenes en la actualidad. Esto con el objetivo de incrementar el índice de satisfacción laboral, la duración dentro de los empleos y la productividad en cada campo de trabajo, ya que estos elementos favorecen al individuo y a su vez al entorno social que se ve beneficiado por jóvenes comprometidos con la economía del país y la ejecución de trabajos formales.

La revisión teórica de las teorías de identidad y la comprensión histórica de los procesos que han llevado a los jóvenes al lugar en el que se encuentran socialmente, darán pistas para gestionar procesos que modifiquen las estructuras sociopolíticas que perpetúan las condiciones de desigualdad en jóvenes de manera general y especialmente en jóvenes mujeres, jóvenes sin acceso a la educación y jóvenes en situación de pobreza.

\section{Agradecimientos}

Agradezco a la SENACYT por la oportunidad de llevar a cabo esta investigación y por el apoyo brindado por parte de los docentes de la Maestría en Ciencias Sociales con énfasis en teorías y métodos de investigación de la Universidad de Panamá. 


\section{Referencias}

Berzonsky, M. (1992). Identity Style and Coping Strategies. Journal of Personality. doi: https://doi.org/10.1111/j.1467-6494.1992.tb00273.x

Burke, P. J., \& Stets, J. E. (2009). Identity Theory. New York: Oxford University Press.

Duque, E., \& Carvajal, L. (2015). La identidad organizacional y su influencia en la imagen: una reflexión teórica. Suma de Negocios, 114-123. doi:https://doi.org/10.1016/j.sumneg.2015.08.011

Freire, M. J. (2009). Los jóvenes y la flexibilidad laboral. Cuadernos de Economía, 5-38. doi:10.1016/S02100266(09)70047-0

Gracia, F., Salanova, M., \& González, P. (1996). La importancia del trabajo en los jóvenes durante los primeros años de empleo. Psicología del trabajo y de las organizaciones, 27-49.

Instituto de Estudios del Trabajo. (2019). UDELAS. Retrieved from Segundo informe sobre la situación del trabajo en Panamá: http://www.udelas.ac.pa/site/assets/files/5613/2o_informe_situacion_del_trabajo_en_pty_final.pdf

Millón, T. (1998). Trastornos de la personalidad. Más allá del DSM-IV. Ed. Masson.

Ministerio de Trabajo y Desarrollo Laboral de Panamá. (2020, Marzo 20). MITRADEL. Retrieved from Panamá presentó un informe sobre la situación de las personas jóvenes que ni estudian ni trabajan: https://www.mitradel.gob.pa/panama-presento-un-informe-sobre-la-situacion-de-las-personasjovenes-que-ni-estudian-ni-trabajan/

Moreno-Hurtado, Torres-Arévalo, Martínez-Patiño, Beltrán, M., \& Vesga-Rodríguez. (2018). Identidad Laboral: Análisis del Concepto en el Contexto Actual del Mundo del Trabajo. Salud y administración, 59-67.

Núñez, Y. (2020). La Estrella de Panamá. Retrieved from Desempleo juvenil, el reto de Panamá en la nueva normalidad: https://www.laestrella.com.pa/cafe-estrella/cultura/200617/desempleo-juvenil-retopanama-nueva

Organización Internacional del Trabajo. (2017). OIT. Retrieved from La débil recuperación del mercado laboral juvenil exige una respuesta radical: https://www.ilo.org/global/about-theilo/newsroom/news/WCMS_598665/lang--es/index.htm

Organizacional Internacional del Trabajo. (2020). OIT. Retrieved from Tendencias mundiales de emplejo juvenil 2020: https://www.ilo.org/wcmsp5/groups/public/---dgreports/---dcomm/--publ/documents/publication/wcms_737662.pdf

Ovejero, A. (2015). Psicología social e identidad: dificultades para un análisis psicosociológico. Papeles del CEIC. doi:http://dx.doi.org/10.1387/pceic.14314

Páramo, P. (2008). La construccion psicosocial de la identidad y del Self. Revista latinoamericana de Psicología, 539-550. doi:0120-0534

Pérez, C., Castro, F., \& Cubo, S. (2009). Significado del trabajo e inserción laboral de graduados universitarios. INFAD Revista de Psicología, 231-246. doi:0214-9877

Pulido-Martínez, H. (2012). La Investigación Sobre la Identidad en, para y por el Trabajo en América Latina, como Ejercicio Crítico Acerca del Mundo Laboral. Psykhe, 77-85. doi:0718-2228

Quinteros, C. (2011). Trabajo, identidad y ciudadanía. Realidad, 261-284. doi:https://doi.org/10.5377/realidad.v0i128.3253 
Romero, M. (2017). Significado del trabajo desde la Psicología del Trabajo. Una revisión histórica, psicológica y social. Psicología desde el Caribe, 120-138. doi:10.14482/psdc.33.2.72783

Ruvalcaba-Coyaso, \& Gutiérrez, U. y. (2011). Identidad e identidad profesional: acercamiento conceptual e investigación contemporánea. CES Psicología, 82-102. doi:https://doi.org/10.21615/1254

Sánchez-Castañeda, A. (2014). Los jóvenes frente al empleo y el desempleo: la necesaria construcción de soluciones multidimensionales y multifactoriales. Revista latinoamericana de Derecho Social, 133162. doi:http://dx.doi.org/10.22201/iij.24487899e.2014.19.9747

Téllez, A. (2002). Identidad socioprofesional e identidad de género. Un caso empírico. Gazeta de Antropología, 1-14. doi:10.30827/Digibug.7400

Zacarés, J., Iborra, A., Tomás, J., \& Serra, E. (2009). El desarrollo de la identidad en la adolescencia y la adultez emergente: una comparación de la identidad global frente a la identidad en dominios específicos. Anales de psicología, 316-329. doi:1695-2294 